\title{
ON $(h-s)_{1,2}$-CONVEX FUNCTIONS AND HADAMARD-TYPE INEQUALITIES
}

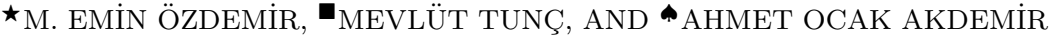

\begin{abstract}
In this paper, two new classes of convex functions as a generalization of convexity which is called $(h-s)_{1,2}-$ convex functions are given. We also prove some Hadamard-type inequalities and applications to the special means are given.
\end{abstract}

\section{INTRODUCTION}

The following definition is well known in the literature [8]: A function $f: I \rightarrow$ $\mathbb{R}, \emptyset \neq I \subseteq \mathbb{R}$, is said to be convex on $I$ if inequality

$$
f(t x+(1-t) y) \leq t f(x)+(1-t) f(y)
$$

holds for all $x, y \in I$ and $t \in[0,1]$. Geometrically, this means that if $P, Q$ and $R$ are three distinct points on the graph of $f$ with $Q$ between $P$ and $R$, then $Q$ is on or below chord $P R$.

Let $f: I \subseteq \mathbb{R} \rightarrow \mathbb{R}$ be a convex function and $a, b \in I$ with $a<b$. The following double inequality:

$$
f\left(\frac{a+b}{2}\right) \leq \frac{1}{b-a} \int_{a}^{b} f(x) d x \leq \frac{f(a)+f(b)}{2}
$$

is known in the literature as Hadamard's inequality (or Hermite-Hadamard inequality) for convex functions. Keep in mind that some of the classical inequalities for means can come from (1.2) for convenient particular selections of the function $f$. If $f$ is concave, this double inequality hold in the inversed way.

Remark 1. 14] Note that the first inequality stronger than the second inequality in (1.2); i.e., the following inequality is valid for a convex function $f$ :

$$
\frac{1}{b-a} \int_{a}^{b} f(x) d x-f\left(\frac{a+b}{2}\right) \leq \frac{f(a)+f(b)}{2}-\frac{1}{b-a} \int_{a}^{b} f(x) d x .
$$

Indeed (1.3) can be written as

$$
\frac{2}{b-a} \int_{a}^{b} f(x) d x \leq \frac{1}{2}\left[f(a)+f(b)+2 f\left(\frac{a+b}{2}\right)\right],
$$

2000 Mathematics Subject Classification. 26D15.

Key words and phrases. h-convex, s-convex, Bullen's inequality.

- : Corresponding Author.. 
which is

$$
\begin{aligned}
& \frac{2}{b-a} \int_{a}^{\frac{a+b}{2}} f(x) d x+\frac{2}{b-a} \int_{\frac{a+b}{2}}^{b} f(x) d x \\
\leq & \frac{1}{2}\left[f(a)+f\left(\frac{a+b}{2}\right)\right]+\frac{1}{2}\left[f(b)+f\left(\frac{a+b}{2}\right)\right] .
\end{aligned}
$$

this immediately follows by applying the second inequality in (1.2) twice (on the interval $\left[a, \frac{a+b}{2}\right]$ and $\left.\left[\frac{a+b}{2}, b\right]\right)$. By letting $a=-1, b=1$, we obtain the result due to Bullen (1978). Further on, we shall call (1.3) as Bullen's inequality.

The inequalities (1.2) which have numerous uses in a variety of settings, has been came a significant groundwork in mathematical analysis and optimization. Many reports have provided new proof, extensions and considering its refinements, generalizations, numerous interpolations and applications, for example, in the theory of special means and information theory. For some results on generalizations, extensions and applications of the Hermite-Hadamard inequalities and convexity, see [1]-[14].

Definition 1. [6] We say that $f: I \rightarrow \mathbb{R}$ is Godunova-Levin function or that $f$ belongs to the class $Q(I)$ if $f$ is non-negative and for all $x, y \in I$ and $t \in(0,1)$ we have

$$
f(t x+(1-t) y) \leq \frac{f(x)}{t}+\frac{f(y)}{1-t} .
$$

Definition 2. [1] We say that $f: I \subseteq \mathbb{R} \rightarrow \mathbb{R}$ is a $P$-function or that $f$ belongs to the class $P(I)$ if $f$ is nonnegative and for all $x, y \in I$ and $t \in[0,1]$, we have

$$
f(t x+(1-t) y) \leq f(x)+f(y) .
$$

Definition 3. 7 Let $s \in(0,1]$. A function $f:(0, \infty] \rightarrow[0, \infty]$ is said to be $s$-convex in the second sense if

$$
f(t x+(1-t) y) \leq t^{s} f(x)+(1-t)^{s} f(y),
$$

for all $x, y \in(0, b]$ and $t \in[0,1]$. This class of $s$-convex functions is usually denoted by $K_{s}^{2}$.

In 1978, Breckner introduced $s$-convex functions as a generalization of convex functions in 4 . Also, in that work Breckner proved the important fact that the set valued map is $s$-convex only if the associated support function is $s$-convex function in [5. A number of properties and connections with s-convex in the first sense are discussed in paper 7 . Of course, $s$-convexity means just convexity when $s=1$.

Definition 4. [9] Let $h: J \subseteq \mathbb{R} \rightarrow \mathbb{R}$ be a positive function. We say that $f: I \subseteq \mathbb{R} \rightarrow \mathbb{R}$ is $h$-convex function, or that $f$ belongs to the class $S X(h, I)$, if $f$ is nonnegative and for all $x, y \in I$ and $t \in[0,1]$ we have

$$
f(t x+(1-t) y) \leq h(t) f(x)+h(1-t) f(y) .
$$


If inequality (1.8) is reversed, then $f$ is said to be $h$-concave, i.e. $f \in S V(h, I)$. Obviously, if $h(t)=t$, then all nonnegative convex functions belong to $S X(h, I)$ and all nonnegative concave functions belong to $S V(h, I)$; if $h(t)=\frac{1}{t}$, then $S X(h, I)=Q(I)$; if $h(t)=1$, then $S X(h, I) \supseteq P(I)$; and if $h(t)=t^{s}$, where $s \in(0,1)$, then $S X(h, I) \supseteq K_{s}^{2}$.

In 1, Dragomir et al. proved two inequalities of Hadamard-type for $P$-functions.

Theorem 1. [1] Let $f \in P(I), a, b \in I$, with $a<b$ and $f \in L_{1}([a, b])$. Then

$$
f\left(\frac{a+b}{2}\right) \leq \frac{2}{b-a} \int_{a}^{b} f(x) d x \leq 2[f(a)+f(b)] .
$$

In 3, Pachpatte established the new following Hadamard-type inequality for products of convex functions.

Theorem 2. 3] Let $f, g:[a, b] \rightarrow[0, \infty)$ be convex functions on $[a, b] \subset \mathbb{R}, a<b$. Then

$$
\frac{1}{b-a} \int_{a}^{b} f(x) g(x) d x \leq \frac{1}{3} M(a, b)+\frac{1}{6} N(a, b)
$$

where $M(a, b)=f(a) g(a)+f(b) g(b)$ and $N(a, b)=f(a) g(b)+f(b) g(a)$.

In 2, Dragomir and Fitzpatrick proved a new variety of Hadamard's inequality which holds for $s$-convex functions in the second sense.

Theorem 3. 20 Suppose that $f:[0, \infty) \rightarrow[0, \infty)$ is an s-convex function in the second sense, where $s \in(0,1)$, and let $a, b \in[0, \infty), a<b$. If $f \in L_{1}([a, b])$, then the following inequalities hold:

$$
2^{s-1} f\left(\frac{a+b}{2}\right) \leq \frac{1}{b-a} \int_{a}^{b} f(x) d x \leq \frac{f(a)+f(b)}{s+1} .
$$

Up until now, there are many reports on convexity and Hadamard-type inequalities. The main purpose of the present paper is to give new classes of convex functions which called $(h-s)_{1,2}$-convex functions as a generalization of ordinary convex functions and to prove new Hadamard-type inequalities for these new classes of functions. Some applications to the special meansof real numbers are given. Throughout this paper we will imply $M(a, b)=f(a) g(a)+f(b) g(b)$ and $N(a, b)=f(a) g(b)+f(b) g(a)$.

\section{New Definitions and Results}

Definition 5. Let $h: J \subset \mathbb{R} \rightarrow \mathbb{R}$ be a non-negative function, $h \neq 0$. We say that $f: \mathbb{R}^{+} \cup\{0\} \rightarrow \mathbb{R}$ is an $(h-s)_{1}$-convex function in the first sense, or that $f$ belong to the class $S X\left((h-s)_{1}, I\right)$, if $f$ is non-negative and for all $x, y \in[0, \infty)=I$, $s \in(0,1], t \in[0,1]$ we have

$$
f(t x+(1-t) y) \leq h^{s}(t) f(x)+\left(1-h^{s}(t)\right) f(y) .
$$

If inequality (2.1) is reversed, then $f$ is said to be $(h-s)_{1}$-concav function in the first sense, i.e., $f \in S V\left((h-s)_{1}, I\right)$. 
Definition 6. Let $\quad h: J \subset \mathbb{R} \rightarrow \mathbb{R}$ be a non-negative function, $h \neq 0$. We say that $f: \mathbb{R}^{+} \cup\{0\} \rightarrow \mathbb{R}$ is an $(h-s)_{2}-$ convex function in the second sense, or that $f$ belong to the class $S X\left((h-s)_{2}, I\right)$, if $f$ is non-negative and for all $u, v \in[0, \infty)=I, s \in(0,1], t \in[0,1]$ we have

$$
f(t u+(1-t) v) \leq h^{s}(t) f(u)+h^{s}(1-t) f(v) .
$$

If inequality (2.2) is reversed, then $f$ is said to be $(h-s)_{2}$-concav function in the second sense, i.e., $f \in S V\left((h-s)_{2}, I\right)$.

Obviously, in (2.2), if $h(t)=t$, then all $s$-convex functions in the second sense belongs to $S X\left((h-s)_{2}, I\right)$ and all $s$-concav functions in the second sense belongs to $S V\left((h-s)_{2}, I\right)$, and it can be easily seen that for $h(t)=t, s=1,(h-$ $s)_{2}$-convexity reduces to ordinary convexity defined on $[0, \infty)$. Similarly, in (2.1), if $h(t)=t$, then all $s$-convex functions in the first sense belongs to $S X\left((h-s)_{1}, I\right)$ and all $s$-concav functions in the first sense belongs to $S V\left((h-s)_{1}, I\right)$, and it can be easily seen that for $h(t)=t, s=1,(h-s)_{1}$-convexity reduces to ordinary convexity defined on $[0, \infty)$.

Example 1. Let $h(t)=t$ be a function and let the function $f$ be defined as following;

$$
f:[2,4] \rightarrow \mathbb{R}^{+}, \quad f(x)=\ln x .
$$

Then $f$ is non-convex and non-h-convex function, but it is $(h-s)_{2}-$ convex function.

The following theorem was obtained by using the $(h-s)_{2}$-convex function in the second sense.

Theorem 4. Let $h: J \subset \mathbb{R} \rightarrow \mathbb{R}$ be a non-negative function, $h \neq 0$. We say that $f: I=[0, \infty) \rightarrow \mathbb{R}$ is an $(h-s)_{2}-$ convex function in the second sense, or that $f$ belong to the class $S X\left((h-s)_{2}, I\right)$, if $f$ is non-negative and for all $x, y \in[0, \infty)=I$, $s \in(0,1], t \in[0,1]$. If $f \in L_{1}[a, b], h \in L_{1}[0,1]$, we have the following inequality:

$$
\frac{1}{b-a} \int_{a}^{b} f(x) d x \leq f(a) \int_{0}^{1} h^{s}(t) d t+f(b) \int_{0}^{1} h^{s}(1-t) d t
$$

Proof. By the definition of $(h-s)_{2}-$ convex mappings in the second sense, for any $s \in(0,1]$ and $t \in[0,1]$, we obtain the following inequality for $u=a, y=b$

$$
f(t a+(1-t) b) \leq h^{s}(t) f(a)+h^{s}(1-t) f(b) .
$$

Integrating both side of (2.4) with respect to $t$ on $[0,1]$, we have

$$
\int_{0}^{1} f(t a+(1-t) b) d t \leq f(a) \int_{0}^{1} h^{s}(t) d t+f(b) \int_{0}^{1} h^{s}(1-t) d t .
$$

Use of the changing variable $t a+(1-t) b=x,(b-a) d t=d x$, we have

$$
\frac{1}{b-a} \int_{a}^{b} f(x) d x \leq f(a) \int_{0}^{1} h^{s}(t) d t+f(b) \int_{0}^{1} h^{s}(1-t) d t
$$

which is the inequality in (2.3). 
Corollary 1. In the inequality (2.3); if we choose $s=1$, we have the inequality;

$$
\frac{1}{b-a} \int_{a}^{b} f(x) d x \leq[f(a)+f(b)] \int_{0}^{1} h(t) d t
$$

Remark 2. If we choose $h(t)=t$, we have the inequality;

$$
\begin{aligned}
\frac{1}{b-a} \int_{a}^{b} f(x) d x & \leq f(a) \int_{0}^{1} t^{s} d t+f(b) \int_{0}^{1}(1-t)^{s} d t \\
& =\frac{f(a)+f(b)}{s+1}
\end{aligned}
$$

which is the right hand side of the inequality in (1.11).

Remark 3. In the inequality in (2.3); If we choose, $h(t)=t$ and $s=1$, we have

$$
\frac{1}{b-a} \int_{a}^{b} f(x) d x \leq f(a) \int_{0}^{1} t d t+f(b) \int_{0}^{1}(1-t) d t=\frac{f(a)+f(b)}{2}
$$

which is the right hand side of the Hermite-Hadamard inequality in (1.2).

Theorem 5. Let $h: J \subset \mathbb{R} \rightarrow \mathbb{R}$ be a non-negative function, $h \neq 0$. We say that $f: I=[0, \infty) \rightarrow \mathbb{R}$ is an $(h-s)_{2}-$ convex function in the second sense, or that $f$ belong to the class $S X\left((h-s)_{2}, I\right)$, if $f$ is non-negative and for all $x, y \in[0, \infty)=I$, $s \in(0,1], t \in[0,1]$. If $f \in L_{1}[a, b], h \in L_{1}[0,1]$, we have the following inequality: $(2.5)$

$$
\frac{1}{2 h^{s}\left(\frac{1}{2}\right)} f\left(\frac{a+b}{2}\right) \leq \frac{1}{b-a} \int_{a}^{b} f(x) d x \leq \frac{f(a)+f(b)}{2} \int_{0}^{1}\left[h^{s}(t)+h^{s}(1-t)\right] d t .
$$

Proof. By the $(h-s)_{2}-$ convexity of $f$, we have that

$$
f\left(\frac{x+y}{2}\right) \leq h^{s}\left(\frac{1}{2}\right) f(x)+h^{s}\left(\frac{1}{2}\right) f(y) .
$$

If we choose $x=t a+(1-t) b, y=t b+(1-t) a$, we get

$$
f\left(\frac{a+b}{2}\right) \leq h^{s}\left(\frac{1}{2}\right) f(t a+(1-t) b)+h^{s}\left(\frac{1}{2}\right) f(t b+(1-t) a)
$$

for all $t \in[0,1]$. Then, integrating both side of (2.6) with respect to $t$ on $[0,1]$, we have

$$
f\left(\frac{a+b}{2}\right) \leq \int_{0}^{1}\left(h^{s}\left(\frac{1}{2}\right) f(t a+(1-t) b)+h^{s}\left(\frac{1}{2}\right) f(t b+(1-t) a)\right) d t .
$$

Use of the changing of variable, we have

$$
\frac{1}{2 h^{s}\left(\frac{1}{2}\right)} f\left(\frac{a+b}{2}\right) \leq \frac{1}{b-a} \int_{a}^{b} f(x) d x
$$

which is the first inequality in (2.5) . 
To prove the second inequality in (2.5), we use the right side of (2.6) and using $(h-s)_{2}$-convexity of $f$, we have

$$
\begin{aligned}
& h^{s}\left(\frac{1}{2}\right)[f(t a+(1-t) b)+f(t b+(1-t) a)] \\
\leq & h^{s}\left(\frac{1}{2}\right)\left[h^{s}(t) f(a)+h^{s}(1-t) f(b)+h^{s}(t) f(b)+h^{s}(1-t) f(a)\right] \\
= & h^{s}\left(\frac{1}{2}\right)\left[h^{s}(t)+h^{s}(1-t)\right][f(a)+f(b)]
\end{aligned}
$$

Integrating the both side of the above inequality,we have

$$
\begin{aligned}
& h^{s}\left(\frac{1}{2}\right) \int_{0}^{1}[f(t a+(1-t) b)+f(t b+(1-t) a)] d t \\
= & h^{s}\left(\frac{1}{2}\right) \frac{2}{b-a} \int_{a}^{b} f(x) d x \\
\leq & h^{s}\left(\frac{1}{2}\right)[f(a)+f(b)] \int_{0}^{1}\left[h^{s}(t)+h^{s}(1-t)\right] d t .
\end{aligned}
$$

We obtain the inequality in (2.5).

Remark 4. In the inequality (2.5); if we choose $h(t)=t$, we have the inequality

$$
2^{s-1} f\left(\frac{a+b}{2}\right) \leq \frac{1}{b-a} \int_{a}^{b} f(x) d x \leq \frac{f(a)+f(b)}{s+1}
$$

which is the inequality (1.11).

Remark 5. If we choose $h(t)=t$ and $s=1$, we have the inequality

$$
f\left(\frac{a+b}{2}\right) \leq \frac{1}{b-a} \int_{a}^{b} f(x) d x \leq \frac{f(a)+f(b)}{2}
$$

which is the Hermite-Hadamard inequality.

Theorem 6. Let $h: J \subset \mathbb{R} \rightarrow \mathbb{R}$ be a non-negative function, $h \neq 0$. We say that $f, g: I=[0, \infty) \rightarrow \mathbb{R}$ are an $(h-s)_{2}-$ convex function in the second sense, if $f, g$ are non-negative and for all $x, y \in[0, \infty)=I, s \in(0,1], t \in[0,1]$. If $f g \in L_{1}[a, b]$, $h \in L_{1}[0,1]$, we have the following inequality;

$$
\begin{array}{ll}
\frac{1}{b-a} \int_{a}^{b} f(x) g(x) d x \leq & f(a) g(a) \int_{0}^{1} h^{2 s}(t) d t+f(b) g(b) \int_{0}^{1} h^{2 s}(1-t) d t \\
& +[f(a) g(b)+f(b) g(a)] \int_{0}^{1} h^{s}(t) h^{s}(1-t) d t .
\end{array}
$$

Proof. Since $f, g \in S X(h-s)_{2}$, we have

$$
\begin{aligned}
f(t a+(1-t) b) & \leq h^{s}(t) f(a)+h^{s}(1-t) f(b) \\
g(t a+(1-t) b) & \leq h^{s}(t) g(a)+h^{s}(1-t) g(b)
\end{aligned}
$$


for all $s \in(0,1], t \in[0,1]$. Since $f$ and $g$ are non-negative,

$$
\begin{aligned}
& f(t a+(1-t) b) g(t a+(1-t) b) \\
\leq \quad & {\left[h^{s}(t) f(a)+h^{s}(1-t) f(b)\right]\left[h^{s}(t) g(a)+h^{s}(1-t) g(b)\right] } \\
= & h^{2 s}(t) f(a) g(a)+h^{s}(t) h^{s}(1-t) f(a) g(b) \\
& +h^{s}(t) h^{s}(1-t) f(b) g(a)+h^{2 s}(1-t) f(b) g(b) .
\end{aligned}
$$

Then if we integrate the both side of the above inequality with respect to $t$ on $[0,1]$, we have the inequality in (2.9).

In the next corollary we will also make use of the Beta function of Euler type, which is for $x, y>0$ defined as

$$
\beta(x, y)=\int_{0}^{1} t^{x-1}(1-t)^{y-1} d t=\frac{\Gamma(x) \Gamma(y)}{\Gamma(x+y)} .
$$

Corollary 2. In the inequality (2.9), if we choose $h(t)=t$ and $s \in(0,1]$, we have

$$
\begin{aligned}
\frac{1}{b-a} \int_{a}^{b}(f g)(x) d x \leq & f(a) g(a) \int_{0}^{1} t^{2 s} d t+f(b) g(b) \int_{0}^{1}(1-t)^{2 s} d t \\
& +[f(a) g(b)+f(b) g(a)] \int_{0}^{1} t^{s}(1-t)^{s} d t \\
= & \frac{M(a, b)}{2 s+1}+N(a, b) \beta(s+1, s+1) \\
= & \frac{M(a, b)}{2 s+1}+N(a, b) \frac{\Gamma^{2}(s+1)}{\Gamma(2 s+2)} .
\end{aligned}
$$

Remark 6. In the inequality (2.9), if we choose $h(t)=t$ and $s=1$, we have

$$
\begin{aligned}
\frac{1}{b-a} \int_{a}^{b}(f g)(x) d x \leq & f(a) g(a) \int_{0}^{1} t^{2} d t+f(b) g(b) \int_{0}^{1}(1-t)^{2} d t \\
& +[f(a) g(b)+f(b) g(a)] \int_{0}^{1} t(1-t) d t \\
= & \frac{M(a, b)}{3}+\frac{N(a, b)}{6}
\end{aligned}
$$

which is the inequality in (1.10).

Theorem 7. Let $h: J \subset \mathbb{R} \rightarrow \mathbb{R}$ be a non-negative function, $h \neq 0$. We say that $f: I=[0, \infty) \rightarrow \mathbb{R}$ is an $(h-s)_{2}$-convex function in the second sense, if $f$ is non-negative and for all $x, y \in[0, \infty)=I, s \in(0,1], t \in[0,1]$. If $f \in L_{1}[a, b]$, $h \in L_{1}[0,1]$, we have

$$
\frac{1}{b-a} \int_{a}^{b} f(x) d x \leq[f(a)+f(b)] \int_{0}^{1} h^{s}(t) d t .
$$


Proof. Since $f \in S X(h-s)_{2}$, by the definition $\left(h-s_{2}\right)$ convexity of $f$, we can write

$$
\begin{aligned}
& f(t a+(1-t) b) \leq h^{s}(t) f(a)+h^{s}(1-t) f(b) \\
& f(t b+(1-t) a) \leq h^{s}(t) f(b)+h^{s}(1-t) f(a)
\end{aligned}
$$

for all $s \in(0,1], t \in[0,1]$. If we add the above inequalities, we write

$$
f(t a+(1-t) b)+f(t b+(1-t) a) \leq[f(a)+f(b)]\left[h^{s}(t)+h^{s}(1-t)\right]
$$

Integrating the both side of the above inequality with respect to $t$ on $[0,1]$, we have

$$
\int_{0}^{1} f(t a+(1-t) b)+f(t b+(1-t) a) d t \leq[f(a)+f(b)] \int_{0}^{1}\left[h^{s}(t)+h^{s}(1-t)\right] d t
$$

By use of the changing of variable and taking into account the $\int_{0}^{1} h^{s}(t) d t=$ $\int_{0}^{1} h^{s}(1-t) d t$ for $s \in(0,1]$, we get

$$
\frac{2}{b-a} \int_{a}^{b} f(x) d x \leq 2[f(a)+f(b)] \int_{0}^{1} h^{s}(t) d t
$$

which completes the proof.

Remark 7. If in (2.10), we choose $h(t)=t$, we have the right hand side of the inequality (1.11). Again, if in (2.10), we choose $h(t)=t$ and $s=1$, we have the right hand side of Hermite-Hadamard inequality. Again, if in (2.10), we choose $h(t)=1$, we have the right hand side of Hermite-Hadamard inequality for P-convex functions in (1.9).

Theorem 8. Let $h: J \subset \mathbb{R} \rightarrow \mathbb{R}$ be a non-negative function, $h \neq 0$. We say that $f: I=[0, \infty) \rightarrow \mathbb{R}$ is an $(h-s)_{2}$-convex function in the second sense, if $f$ is non-negative and for all $x, y \in[0, \infty)=I, s \in(0,1], t \in[0,1]$. If $f \in L_{1}[a, b]$, $h \in L_{1}[0,1]$, we have

$$
\frac{1}{b-a} \int_{a}^{b} f(x) d x \leq\left[\frac{f(a)+f(b)}{2}+f\left(\frac{a+b}{2}\right)\right] \int_{0}^{1} h^{s}(t) d t .
$$

Proof. By the $(h-s)_{2}-$ convexity of $f$, we have

$$
\begin{aligned}
& f\left(t a+(1-t) \frac{a+b}{2}\right) \leq h^{s}(t) f(a)+h^{s}(1-t) f\left(\frac{a+b}{2}\right) \\
& f\left(t \frac{a+b}{2}+(1-t) b\right) \leq h^{s}(t) f\left(\frac{a+b}{2}\right)+h^{s}(1-t) f(b)
\end{aligned}
$$

If we integrate the both side of the above inequalities with respect to $t$ on $[0,1]$, and use of the changing of variable, we get

$$
\frac{2}{b-a} \int_{a}^{\frac{a+b}{2}} f(x) d x \leq f(a) \int_{0}^{1} h^{s}(t) d t+f\left(\frac{a+b}{2}\right) \int_{0}^{1} h^{s}(1-t) d t
$$

and

$$
\frac{2}{b-a} \int_{\frac{a+b}{2}}^{b} f(x) d x \leq f\left(\frac{a+b}{2}\right) \int_{0}^{1} h^{s}(t) d t+f(b) \int_{0}^{1} h^{s}(1-t) d t .
$$


By adding the above inequalities and taking into account the $\int_{0}^{1} h^{s}(t) d t=\int_{0}^{1} h^{s}(1-t) d t$ for $s \in(0,1]$, we get

$$
\frac{2}{b-a} \int_{a}^{b} f(x) d x \leq\left[f(a)+f(b)+2 f\left(\frac{a+b}{2}\right)\right] \int_{0}^{1} h^{s}(t) d t
$$

which completes the proof.

Corollary 3. If in (2.11), we choose $h(t)=t$, we have

$$
\frac{1}{b-a} \int_{a}^{b} f(x) d x \leq \frac{1}{s+1}\left[\frac{f(a)+f(b)}{2}+f\left(\frac{a+b}{2}\right)\right] .
$$

Remark 8. If in (2.11), we choose $h(t)=t$ and $s=1$, we have

$$
\frac{1}{b-a} \int_{a}^{b} f(x) d x \leq \frac{1}{2}\left[\frac{f(a)+f(b)}{2}+f\left(\frac{a+b}{2}\right)\right]
$$

which is the inequality (1.4).

\section{Applications to Some Special Means}

We now consider the applications of our Theorems to the following special means

b) The arithmetic mean:

$$
A=A(a, b):=\frac{a+b}{2}, \quad a, b \geq 0,
$$

c) The geometric mean:

$$
G=G(a, b):=\sqrt{a b}, \quad a, b \geq 0,
$$

d) The harmonic mean:

$$
H=H(a, b):=\frac{2 a b}{a+b}, \quad a, b \geq 0,
$$

e) The quadratic mean:

$$
K=K(a, b):=\sqrt{\frac{a^{2}+b^{2}}{2}} \quad a, b \geq 0,
$$

f) The logarithmic mean:

$$
L=L(a, b):=\left\{\begin{array}{ll}
a & \text { if } a=b \\
\frac{b-a}{\ln b-\ln a} & \text { if } a \neq b
\end{array}, \quad a, b \geq 0,\right.
$$

g) The Identric mean.

$$
I=I(a, b):=\left\{\begin{array}{ll}
a & \text { if } a=b \\
\frac{1}{e}\left(\frac{b^{b}}{a^{a}}\right)^{\frac{1}{b-a}} & \text { if } a \neq b
\end{array}, \quad a, b \geq 0,\right.
$$

h) The $p$-logarithmic mean:

$$
L_{p}=L_{p}(a, b):=\left\{\begin{array}{ll}
{\left[\frac{b^{p+1}-a^{p+1}}{(p+1)(b-a)}\right]^{1 / p}} & \text { if } a \neq b \\
a & \text { if } a=b
\end{array}, \quad p \in \mathbb{R} \backslash\{-1,0\} ; \quad a, b>0 .\right.
$$


The following inequality is well known in the literature:

$$
H \leq G \leq L \leq I \leq A \leq K
$$

It is also known that $L_{p}$ is monotonically increasing over $p \in \mathbb{R}$, denoting $L_{0}=I$ and $L_{-1}=L$.

The following propositions holds:

Proposition 1. Let $a, b \in(2, \infty), a<b$. Then for all $s \in[0,1]$, we have

$$
\ln I(a, b) \leq \frac{2}{(s+1)} A(\ln a, \ln b)
$$

Proof. The proof is obvious from Theorem 4 applied $f(x)=\ln x, h(t)=t, x \in$ $[2, \infty)$ and $s \in[0,1]$.

Proposition 2. Let $a, b \in(2, \infty)$. Then for all $s \in[0,1]$, we have

$$
2^{s-1} \ln (A(a, b)) \leq \ln I(a, b) \leq \frac{1}{(s+1)} A(\ln a, \ln b)
$$

Proof. The assertion follows from Theorem 5 applied for $f(x)=\ln x, x \in[2, \infty)$ and $h(t)=t$.

Proposition 3. Let $a, b \in(2, \infty)$. Then for all $s \in[0,1]$, we have

$$
\ln I(a, b) \leq \frac{1}{(s+1)} \ln G^{2}(a, b)
$$

Proof. The prof is immediate follows from Theorem 7 applied for $f(x)=\ln x$, $x \in[2, \infty)$ and $h(t)=t$.

Proposition 4. Let $a, b \in(2, \infty)$. Then for all $s \in[0,1]$, we have

$$
\ln I(a, b) \leq \frac{1}{(s+1)}\left[\frac{\ln G^{2}(a, b)}{2}+\ln (A(a, b))\right]
$$

Proof. The prof is immediate follows from Theorem 8 applied for $f(x)=\ln x$, $x \in[2, \infty)$ and $h(t)=t$.

\section{REFERENCES}

[1] Dragomir, S.S., Pečarić, J. and Persson, L.E. Some inequalities of Hadamard type, Soochow J.Math., 21, 335-341, 1995.

[2] Dragomir S.S. and Fitzpatrick S., The Hadamard's inequality for $s$-convex functions in the second sense, Demonstration Math., 32 (4) (1999), 687-696.

[3] B.G. Pachpatte, On some inequalities for convex functions, RGMIA Res. Rep. Coll., Vol. 6, Art. 1 (2003) 1-9.

[4] Breckner, W.W. Stetigkeitsaussagen f"ur eine Klasse verallgemeinerter konvexer funktionen in topologischen linearen Raumen, Pupl. Inst. Math., 23, 13-20, 1978.

[5] Breckner, W. W. Continuity of generalized convex and generalized concave set-valued functions, Rev Anal. Num'er. Thkor. Approx., 22, 39-51, 1993.

[6] Godunova, E.K., Levin, V.I. Neravenstva dlja funkcii sirokogo klassa, soderzascego vypuklye, monotonnye $i$ nekotorye drugie vidy funkii, Vycislitel. Mat. i. Fiz. Mezvuzov. Sb. Nauc. Trudov, MGPI,Moskva, pp. 138-142, 1985.

[7] Hudzik, H., Maligranda, L. Some remarks on s-convex functions, Aequationes Math., 48, 100-111, 1994.

[8] Mitrinović, D.S., Pečarić, J. and Fink, A.M. Classical and new inequalities in analysis, KluwerAcademic, Dordrecht, 1993.

[9] Varošanec, S. On h-convexity, J. Math. Anal. Appl., Volume 326, Issue 1, 303-311, 2007. 
[10] Bombardelli, M., Varošanec, S. Properties of $h$-Convex functions related to the HermiteHadamard-Fejér inequalities, Comput. Math. Appl. 58 (2009) 1869-1877.

[11] Burai, P., Hazy, A. On approximately h-Convex functions, J. Convex Anal. 18 (2) (2011) 447-454.

[12] Sarıkaya, M.Z., Sağlam, A., Yıldırım, H. On some Hadamard-type inequalities for $h-$ Convex functions, J. Math. Inequal. 2 (3) (2008) 335-341.

[13] Özdemir, M.E., Gürbüz, M. and Akdemir, A.O. Inequalities for $h$-Convex Functions via Further Properties, RGMIA Research Report Collection Volume 14, article 22, 2011.

[14] S.S. Dragomir, C.E.M. Pearce, Selected topics on Hermite-Hadamard inequalities and applications, RGMIA monographs, Victoria University, 2000. [Online: http://www.staff.vu.edu.au/RGMIA/monographs/hermite-hadamard.html].

* Atatürk University, K. K. Education Faculty, Department of Mathematics, 25240, Campus, Erzurum, Turkey

E-mail address: emos@atauni.edu.tr

- Kilis 7 Aralik University, Faculty of Science and Arts, Department of MathematICS, 79000, Kilis, TURKEY

E-mail address: mevlutunc@kilis.edu.tr

- AĞRi İbrahim Çeçen University Faculty of Science and Letters, Department of Mathematics, 04100, AĞRi, Turkey

E-mail address: ahmetakdemir@agri.edu.tr 